\title{
Space Exploration: Approaches to Inhabiting Digital Spaces and Their Influence on Education
}

\author{
Nigel Calder ${ }^{1}$ (D) Kathrin Otrel-Cass ${ }^{2}$ iD
}

Accepted: 18 October 2020 / Published online: 10 November 2020

(C) The Author(s) 2020

\begin{abstract}
What happens when we go online, interact and leave our digital footprints? What is the nature of the online spaces that teachers and their students inhabit and the implications of being in these spaces? In this article we have explored these questions by following the theoretical inspirations by James Paul Gee about affinity spaces and Martin Heidegger's notions on dwelling. The article interweaves its argument with examples from several research projects to argue that online environments allow for opportunities to play and personalize, to be creative, and that these forms of expressions are an interplay of social and technical elements. While the control within digital spaces is not transparent, we contend that there are opportunities for the user to exert influence on and within digital spaces, and to transform them in varying ways and scope. Sometimes those spaces facilitate autonomy and self-selection, which in turn initiates or confirms transformation. With the growth and increased sophistication of virtual realities and artificial intelligence, we need to understand the nature of the educational engagement within these spaces. We also need to understand this mutually influencial engagement between the user and these digital spaces, and be vigilant as to who might be exerting the most influential control.
\end{abstract}

Keywords Digital technologies · Online environments · Dwelling · Apps · Affinity spaces

\section{Introduction}

Digital technologies, and mobile technologies in particular, open up new spaces for learners to engage with ideas and processes. Simultaneously, they offer opportunities for the learning situation to unfold in alternative ways from more traditional

Kathrin Otrel-Cass

kathrin.otrel-cass@uni-graz.at

1 University of Waikato, Hamilton, New Zealand

2 University of Graz, Graz, Austria 
pedagogical media such as pen-and-paper (e.g., Calder and Murphy 2018; Gee 2005). These new media spaces are shaped by (digital) material characteristics as well as the specific practices they afford their users. When used for educational purposes, a rethinking on how to incorporate them appropriately into teaching approaches is required, since they potentially enable greater flexibility and open-ended interaction for their users.

Learning processes in general appear to manifest more as a coalition of material and social entities, or what Meyer (2015) termed a socio-material bricolage. Others (e.g., Calder and Murphy 2018) also acknowledge this interplay of material and social elements but have included the significance of participant worldviews, and the associated discourses that lead participants to this positioning. This resonates with a postdigital perspective, where assemblages of digital, material and social entities might facilitate more nuanced, personalized learning pathways, with the distinction between physical and digital pedagogical media becoming less distinct (Calder and Murphy 2018). They contend that the participants' prevailing worldviews will influence the nature of the interactions with both the digital technology and the social fabric within which it is enacted. This interaction will in turn influence the participants' perspectives. In a similar way it has been argued that engagement within a digital medium is influenced by the affordances of the digital medium, but this engagement in turn influences the nature of the digital medium (Hoyles and Noss 2003). Both the digital medium and the user are mutually influential, and influenced through the interaction with each other. The participants' worldviews might therefore be considered part of the social fabric of this coalition of material and social entities.

Allied to this, Gee (2005) contends that digital technologies open up 'affinity spaces' for learning, locations where groupings congregate through a shared interest, or engagement with a shared activity. He articulated these affinity spaces as being '... a place or set of places where people affiliate with others based primarily on shared activities, interests, and goals, not shared race, class culture, ethnicity, or gender' (67). For Gee (2005), what is happening in online cultures is different from offline communities. From Gee's perspective, the word 'community' evokes a sense of belonging and membership. However, he has envisaged these worlds rather as 'spaces'-a term that allows for the ongoing ebbs and flows of engagement by various participants and with differing intensities of involvement and participation demonstrated by different members.

Wenger's $(1998,2010)$ description of communities of practice defines at the core communities that build and dissolve depending on their interest needs leaving material traces of their coming together. Wenger notes:

Engagement and social context involves a dual process of meaning making. On the one hand, we engage directly in activities, conversations, reflections, and other forms of personal participation in social life. On the other hand, we produce physical and conceptual artifacts - words, tools, concepts, methods, stories, documents, as to resources, and other forms of reification - that reflect our shared experience and around which we organize our participation. (Literally, revocation means making into an object). (Wenger 2010: 180)

Meanwhile, there are digital spaces that children, in fact people generally, inhabit; where they are drawn to congregate in a communal sense and those where they might 
just visit. For instance, I might engage within an online community such as a social network that I perhaps inhabit over a long period of time. This habitation might ebb and flow dynamically in terms of content and participation, and here I might be leaving my digital 'footprints'. On the other hand, I might visit a news or weather online location as a visitor rather than as a contributing participant. This kind of inhabitation can be likened to what Ingold (2005: 502) describes as 'weaving a path through a medley of structures built by others for you to live in'. Heidegger explains that: 'The relationship of people to locations, and through locations to spaces is based on dwelling. The relationship between human and space is none other than dwelling...' (Heidegger 1951: 12, italics in original). This quotation of Heidegger's work is one we want to return to unpack further to help explain our interpretation of the notion of dwelling and inhabiting digital spaces.

In this paper we will try to examine digital spaces as dwellings that we might inhabit, as defined by Heidegger (1951), to better understand their nature and how they might differ from the spaces that we visit only, to gain insights into the opportunities and constraints that these spaces afford and whether these affordances evoke and sustain habitation. We will also consider the nature of personalization, and ownership that might determine the difference between the spaces we inhabit rather than visit. To personalize something suggests that there is an opportunity to modify something, so that it is identified with or comes from a particular person. It implies the possibility to be inquisitive and play with given possibilities. In this regard, personalized learning might be seen as learning that is personally relevant (Willacy et al. 2017).

Firstly, we consider the meaning of affinity spaces and how these might be influential in creating dialogical spaces with an opportunity for more enhanced versions of learning and identity to emerge. Throughout the paper we use vignettes from selected research studies and associated experiences. From Spalding and Phillips (2007) we have learned that vignettes can be used to "provide a mediated account of the "truth" (providing the data collection methods are also trustworthy)' (961). The vignettes were written specifically to explain our analysis and support meaning. They are accounts we produced in reflection of the collected and analysed data. Taking this approach enabled us to draw on an eclectic array of types and purposes of research studies while also allowing us to more specifically illustrate particular points. This kind of vignette presentation has been described as composites since we did not mean to draw our insights from any single observation. However, different to Spalding and Philips we did not draw different events together in one fictionalized account but wanted to make clear to the reader that these accounts stem from different studies allowing us as authors to bring in our own voices and the portrays of the different .participants we were working with.

\section{Places to Dwell}

To better understand what we mean by places to dwell we begin by examining the meaning of affinity spaces and how these might be influential in creating dialogical spaces. We will illustrate that dialogic spaces can offer an opportunity for more enhanced versions of learning and identity to emerge, especially when the 'neediness' of one participant shapes the other' through his dialogue to identify 'personal participation, perspectives, evaluations or positions' (Otrel-Cass and Andrule 2015: 140). We also consider locations. Finally, we turn to Heidegger's ideas on dwelling. 


\section{Affinity Spaces}

In affinity spaces people assemble in congregation based on their shared common interests, pursuits or activities (Gee 2005). They are also spaces that might initiate and encourage sharing and participation. While indicating that these spaces usually occur online, Gee does, however, maintain that they can be physical or virtual. However, Gee (2005) sees these spaces as different from a community, which he contends has connotations of formal membership and belonging (to the community). This differentiation of spaces from community is in recognition of the 'robust characterization of the ebbs and flows and differing levels of involvement and participation exhibited by the members' (Gee 2005: 70). Yet despite this, and the ease with which people can participate in online communities of various forms, these spaces usually have a sign up page that collects personal information. What are the underlying political and economic discourses that might sit behind this? Yet, these also are relatively easy to subvert, with people able to create multiple identities and avatars that might conceal their true identity or conversely allow them to reveal their real identity but in a way that will not be recognized.

Vignette 1: My avatar: In the study 'One day in my onlife' that was conducted between 2017 and 2020 (see for example Otrel-Cass 2019) young people between the ages of 16 and 28 were interviewed about their online practices and asked to share one day of online activities. I asked Mona (pseudonym), 16 years old, why she had created an avatar (see Fig. 1):

Mona: You can send them to each other and you can send them also interacting with each other, you can use them as a reaction. I think most people use them ironically.

R: When you create your avatar, do you try and be correct or do you invent something about yourself?

Mona: I think most people create an avatar which is generally genuinely accurate to how they look but you can, I mean you can make yourself a blue dragon head or something and I don't think people care about them too much, but I guess this is what most people do that I know.

Me: So why would you not take a photo of you instead?

Mona: Well the ones I use are emojis, I use them on Snapchat which is like a photo but I can send the avatar and add them as stickers, so you can add 'save my avatar' for someone else's editor so we can just send them over a chat and that can be stupid or funny.

Me: So, can the avatars do things?

Mona: Oh yes, they're can be in different positions or something, not just a smiley face (see Fig. 2). I mean you can just send a smiling one, but I mean you can also send some with like hot eyes on somebody telling a joke or whatever. It's almost like sending a meme or a gif, except it's yourself and perhaps another person instead of just sending a random photo. 


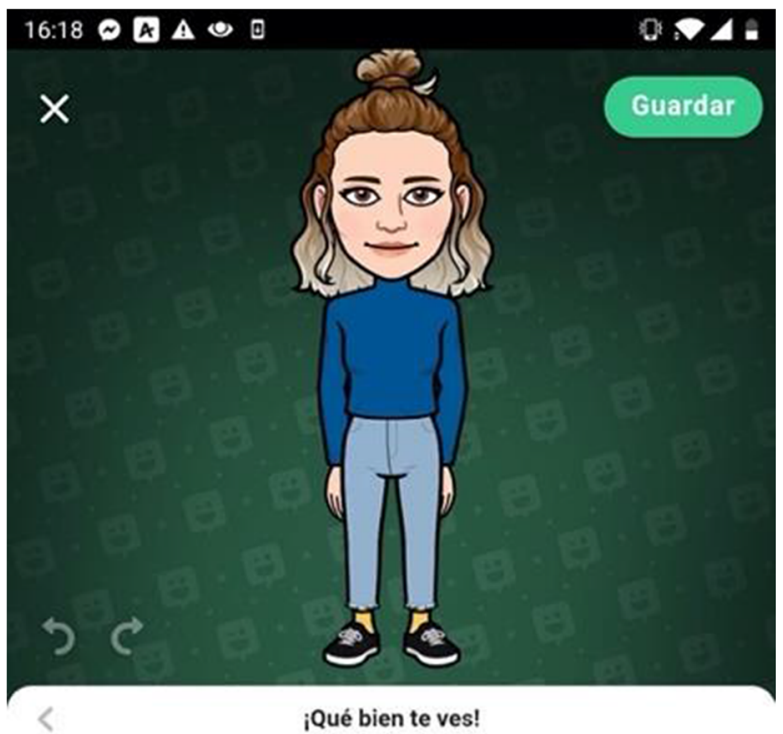

\section{Guardar avatar}

Fig. 1 Mona's avatar

Mona explains that this avatar is usually based on her own identity, not necessarily to conceal but to personalize and add an emotive dimension to her communications with her friends. Mona personalizes selected online spaces through her avatars. Her interactions carry a degree of emotive element with it. In these spaces the avatars seem to be used to (over)emphasize her dialogic interactions with her friends.

According to Gee (2005), spaces in general are defined by three distinguishing attributes. First, a space needs content - an aspect that the space is concerned with. In the spaces that we are concerned with this might be about conceptual understanding, opinions or social engagement. Each space also requires what he terms a generator. Generators create the content for the space. This might be teachers, students, digital media, phenomena and the Internet, all of which could in various ways act as generators. Spaces also need at least one portal according to Gee. Portals are places 


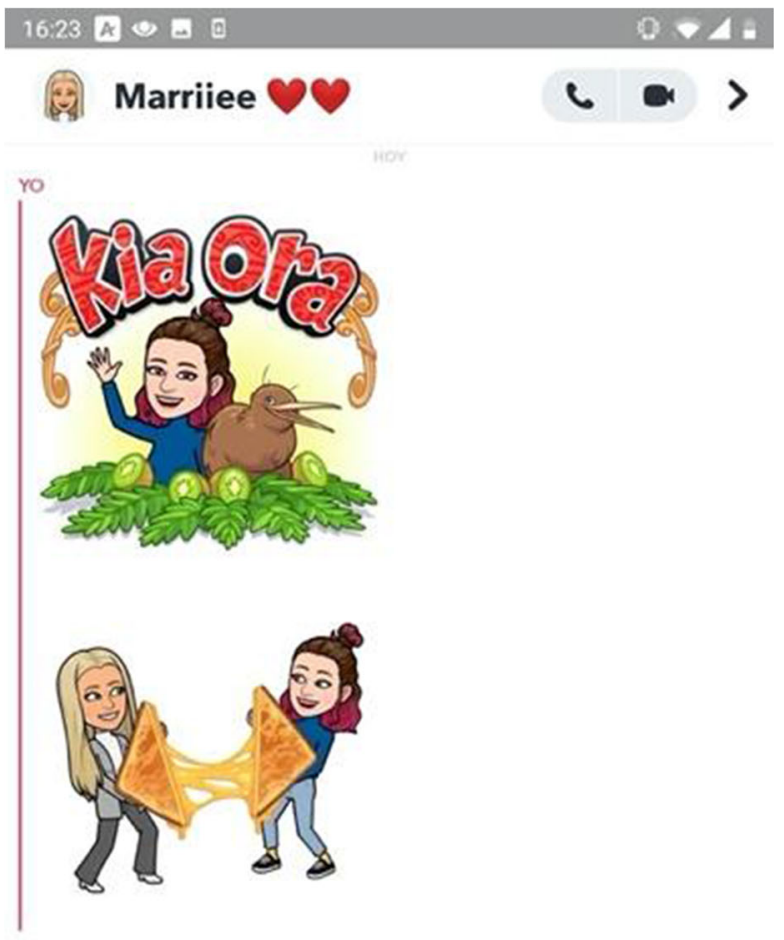

Fig. 2 Mona's avatar sharing pizza with a friend's avatar

through which people or programmes such as search engines access content. In a real environment the classroom door can be considered as a portal, while the Instagram app acts as a virtual portal for its users to enter content.

It is common for generators to also be portals and vice versa (Gee 2005). For instance, an Internet site might both generate content and be the portal to access that content. Using the three attributes: content, generator and portal, helps to unpack the nature of space and how it is defined through its content, as well as how people generate, access and interact with that content. The examination of these three features also enables us to determine the extent to which a space can be an affinity space. 
Affinity spaces are spaces, real or virtual where people bond primarily to a shared endeavour. They represent spaces with shared intentionality.

Affinity spaces are considered particularly important contemporary social configurations, which educators ought to attend to for two main reasons (Gee 2005). Firstly, he contends that affinity spaces have features, which are crucial for deep and effective learning, by which he means learning which has a lasting impact on identity and ability to participate in desired discourses. Secondly, that the current proliferation of affinity spaces young people can access offers them 'a different and arguably powerful vision of learning, affiliation, and identity' (Gee 2005: 29) where learning is simultaneously a personal, unique trajectory and a social journey as one shares aspects of that trajectory with others. His suggestion is to make classrooms and learning spaces more characteristic of affinity spaces, in order to promote deep individual and social learning through students bonding with the learning endeavour.

Vignette 2: Sharing online across the globe: In the Networked Inquiry in Learning Secondary Science (NILSS) study (see for example Williams et al. 2013) students from New Zealand communicated with students in Denmark using the platform Padlet (www.padlet.com). The students' task was to share some of their questions and information they had on a broad astronomy topic. They left posts and replied at times to each other's posts (see Fig. 3). The students left several posts from over a few weeks. They posted questions and responded to each other. Interestingly, the students chose to use Google translate to communicate with each other.

For example, Sam from New Zealand writes: Do you want me typing in Danish or English? And translates it further to: Vil du have mig at skrive på dansk eller engelsk? He replies to a number of posts by the Danish students Emil and Cyrus and receives a reply to one of his own questions from Sebastian from Denmark.

In this example we find that the coming together of the online space combined with the learning task and written enactment of the students created the conditions for an affinity

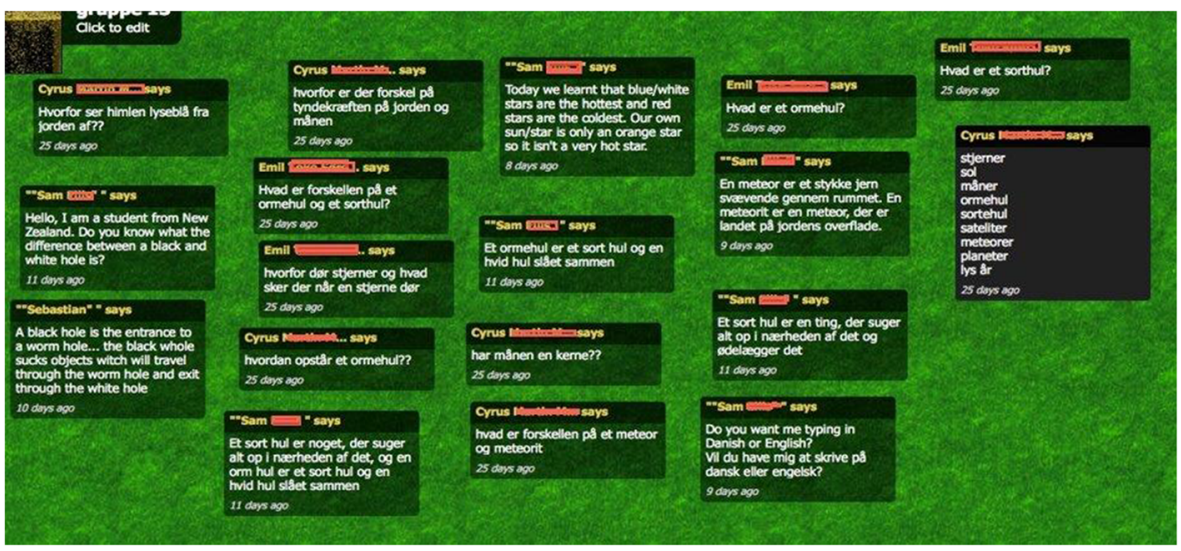

Fig. 3 Padlet page from NILSS project 
space. The unique URL assigned to the Padlet page was only available to the group of students and not the entire class. The written conversations amongst each other were not simply posts of knowledge sharing but indicated intentionality, perhaps however, the intention was to get to know and learn about each other, for instance through exploring the different languages they spoke. The group came and worked together since their teachers had assigned them to a task, their shared intentionality was to some degree shaped by what the teachers had asked them to do and also by their curiosity to communicate with someone from the other side of the globe. This was a comment that the teachers told us several times. They told us that this made "being in that space" interesting, exciting and sometimes frustrating (for example, when students had to wait too long for responses arriving from the other side), was the interaction albeit asynchronously with each other. The Padlet that was shared between the group in New Zealand and Denmark documented a personal, unique trajectory and a social journey between the students. It set the conditions to promote individual and social learning. The Padlet technology per se was not the affinity space but rather it set the conditions to create a very unique environment. The resulting product evidenced the presence of an affinity space.

Both Heidegger (1951) and Gee (2005) articulated perspectives of spaces (whether concrete or digital), which are defined by and hinged to the people who visit them and might stay and interact within them for varying periods of time. It is thus the people and their activity that shape the nature and type of dwelling that occurs in the space.

One aspect that Heidegger and Gee do not appear to demonstrably align is the element of content. Gee contends that content is a key feature of space that needs to be generated so it can initiate engagement or occupation. The space needs to be about something so that people will congregate there, it is what their affiliation is based on. However, in Heidegger's version of space, although people are also essential to the defining milieu, the location rather than the content is the key element. Of course, there are a range of interplays between locations and content; hence, they are not mutually exclusive. But this delineation might lead to differences in the nature of spaces and the relationships that evolve within them; hence, the nature of dwelling might differ. Likewise, the affordances of the spaces or their influencing elements might also be different, both for material and socialcultural elements of the space. However, we return again to Heidegger's focus on the defining nature of relationship since: 'The relationship between human and space is none other than dwelling' (1951: 12).

\section{Collective Dwelling and Revealing Ourselves: Dwelling and Aesthetics}

Ingold (2005: 503) notes that 'the trouble with dwelling is that it sounds too cozy' and points out that we should remember that dwelling is also about living together and that dwelling is essentially a collective activity. Inhabited collectives are shaped by 'fields of power' (Ingold 2005: 503) and thus indicate the existence of political dimensions that are fashioned by the values societies attribute to those constructions. It is through the production of ourselves in space where we create content with intentions. Heidegger explains that there are poetic and technological modes of revealing ourselves through such constructs and when we interpret these constructs, we should realize the inner qualities of the materials used for our constructions, meaning the decisions and interpretations that have been encapsulated. Thus, the way 
we dwell is also an expression of our views and interpretations on aesthetics and beauty (Richter 1995). Inhabitation may then indicate intentionality and appreciation of the relationship between spaces and people who dwell there.

How might this manifest in virtual locations? Take, for example, digital locations that were built but the intended purpose is not or no longer applicable. Perhaps software that does not function effectively on mobile technologies such as smart phones or hardware such as desktop computers. And what happens when the intended or original users move to an alternative location? Perhaps the 100 million global users of Myspace who are no longer there but have moved to alternatives such as Facebook or Instagram. As well, a range of aspects as simple as fashion, aesthetics and functionality can influence the use of apps or social networks. If an app is aesthetically pleasing in appearance or has enhanced functionality, perhaps being simpler or more intuitive to use than another app that has the same content or purpose, then it will most likely be more popular. The heterogeneity between humans and the digital environment can lead to people producing aesthetic stories in online spaces, to produce a world according to people's imagination which are not necessarily depictions of the real world (Welsch 2014). Both aesthetics and functionality relate to fashion, although as with clothes, something might be more fashionable with a particular community or cohort without being either more functional or aesthetically pleasing. Each of those aspects is a varying blend of social and technical elements. If all falls into place, we might dwell and inhabit these spaces and produce content.

So, what might be the affordances of places that are inhabited rather than visited? While affordances inherently are entangled with the social fabric in which the user exists; what Gibson (1977) termed the complementarity of the user and the environment, let us first consider some material elements while assuming the social milieu in which they exist and are mutually influential with. Social media, or at least some versions of it, seem to be places that users inhabit. For instance, Facebook has 3.9 billion users and 1.47 billion of them use it daily. One key element of social media such as Facebook is the ease of connectivity with a group called 'friends' that are self-selected. The connectivity is a material element but to whom you actually connect is the related social element.

This would seem a manifestation of an afinity space, as would a blog that is focused around an area of interest or contestation. A common attribute of both is that the user can edit their contributions according to their aesthetic preferences, bound by the functionality of the system, before submitting them. Also, these contributions are then held and accessible for a period of time. They are relative stable entities, which is part of their materiality. The following vignette considers some of these attributes and the aspect of self-selection.

Vignette 3: What features of apps do you like? This vignette is data from a series of teacher semi-structured interviews from teacher co-researchers in research projects examining the use of apps to learn mathematics. They were considering their reasons for choosing apps for their personal use. The excerpts used were indicative of the overall responses. The first excerpts highlight the social aspect 
with the participant using the app to maintain relationships over distance and share their opinions and insights. It was in response to the question: What features of apps do you like?

Dana: Keeping in touch with friends and following what they have been up to recently. Following celebrities and people I find inspirational and getting a glimpse into their lives. The opportunity to share your opinions. Seeing photos related to my hobbies or places I have travelled to or am interested in.

Mike: That they're pretty easy to navigate. I like apps where I can share things because then we can share it - so probably apps that are a little bit collaborative.

Meanwhile, some particular apps or websites have specific material aspects. Twitter for instance limits a tweet to 280 characters, while Instagram is structured more purposefully around photo or video posts. These features have ramifications for the social nature of the engagement and perhaps the age and characteristics of the users. Importantly, they have been designed for particular potential users or adapted as the relationship between the technology and the user has stabilized or evolved. For instance, as the use of Twitter became more prevalent for political and academic comment, the need for more characters became necessary, hence the change from a limit of 140 to 280 characters. Interestingly, all participants directly mentioned using some apps regularly but for short intervals due to the content in particular, but also due to the features of the functionality of the related apps.

Rene: The apps that I use most often, for a long period of time, in one single session would be stuff - which I would have no community with but it has a lot of content for me that I devour. I use stuff just for reading the news. With a banking app it's the ease of transferring money and getting information. I wouldn't spend a lot of time there. More just do the business and then move on.

Anaru: Things like google map type apps to find places. Not that often. Things like that when I have a particular purpose. So, things like banking apps checking and paying bills and things like that I would check that probably most days. I have an app for the fitness studio that I belong to. I would go on there most days to book a class or to cancel a class.

Interviewer: What features of each of these do you find useful?

Dana: They are easy to peruse and to skip stories, posts etc. that you are uninterested in, you have a lot of control over the type of content you see. Interviewer: What are aspects of apps that put you off using them?

Dana: When the login process takes too long, too many advertisements, apps that take too long to navigate and find what you're looking for, apps that make it hard to learn about or control your privacy settings, apps that make it too complicated to delete or deactivate your account. 
Ria: So, you don't want to have to read a manual to work it. You want to be able to just muck around with it and say this is how this works so I'm good to go intuitively easy to navigate.

Rene: Too much advertising, bad functionality, needing an account and sign in to use them and cost.

Interviewer: What are features of the apps that affect the way you use them?

Dana: Thumb ID for quick and easy logins, having pictures or symbols that help you to quickly find content that is relevant or of interest to you.

Anaru: They're easy to get on to; they perform a quick purpose and I can sort of get on with life. That's what a good app is for me.

Rene: It's just the usability of them. The ones that are easier to use are more functional.

Dana: Quick login process, quick and simple navigation, engaging content.

Ria: Free, easy to use, if friends are using them or they serve a purpose.

These data indicate elements of both functionality and purpose. A common thread is the user's perceived control, having choices and individualizing the space. This also suggests a desire to manage their digital footprint.

The materiality of social media includes a stability of text, images and sound, what Treem and Leonardi (2012) have termed persistence. This means that one's posts are stowed in the system where they remain while being accessed later and intermittently (Binder, Howes, and Sutcliffe 2009). But editability and persistence are not specific to social media. For instance, email and blogs offer a high degree of editability. What is different though is the public nature of the posts. Social media differ from technologies like email because their materiality enables people's posts to be immediately broadcast to a large unknown audience (Treem and Leonardi 2012). This makes their posts visible beyond say one or two intended users. This difference in materiality means that people who use social media have to recognize that their posts, comments, tweets and questions are public. To appreciate the consequences of this, the nature of this visibility and the conditions under which it occurs, we need to recognize that the technology has a materiality that permits certain actions while making others impossible, or at least constraining their enactment (Treem and Leonardi 2012). This materiality indicates further affordances of the media: the structure and features of the platform where the posts, comments, tweets and questions exist, and how the user and others engage with these, that is, the connectivity with other users.

Within open environments, locations are defined by the entities that are constructed there. Heidegger (1951) discussed this in terms of the metaphor of a river. There are many potential locations on a river for a bridge to be built, although some will offer better opportunities for construction and also to enhance its use. They will all have some constraints. Where the bridge is situated determines a location. It is from the bridge itself that a location is made (Heidegger 1951). In concrete terms a building might be constructed with the intention to become a dwelling, but may still not become one even if it is undertaken for that purpose. This may be for a range of reasons. It may be that the intended purpose for the building is no longer applicable, it may be that those for whom the building was intended are now in an alternative location, or it may be the socio-cultural discourse associated with the building is inconsistent with that of the intended or potential subsequent users. For instance, a post office in a small village 
may once have been a key element of the village's connectivity outside of the village, and essential for communication and the local economy. With changes to communication technology this purpose is no longer applicable. The shops, schools and houses in an area close to a coal mine may no longer be inhabited as alternative energy resources to coal, such as wind and nuclear became more prevalent and workers and their families moved away when the coal mine closed down. Likewise, two restaurants might have similar quality premises and food, but one is popular and one not because of the style of food (say, Italian cf. Indian) or even if the food is also the same the conviviality of the staff or reputation of the owner may determine whether it is frequented or not. Hence, both dwelling and visiting are contingent on more than the attributes of the physical or digital entity.

Similarly, in digital environments, it is from the construction of an app, or social or virtual network that a location is designated. Through this perspective though, locations that are intended to be inhabited in some way are also inherently related to those that might use or inhabit them. Spaces are not discrete entities from people. They do not exist as spaces without a relationship with people. A space is not something that is positioned contrary to people, nor is it an external entity or an inner encounter. 'There are not humans and in addition to them space' (Heidegger 1951: 11). But having digital spaces created even with identification of the potential users does not ensure its use or the sustainability of its use. The affiliation of the potential users of that digital space with the space is through dwelling. Here we return to Heidegger's general contention of this relationship as: 'The relationship between human and space is none other than dwelling' (12). So, it is fair to conclude that just as the human condition, and broader and individual social elements, are in transition, and might evolve or at least vary through time, and the nature and scope of the influencing experiences, so too will the nature of dwelling transition or vary. At times the aim and intention for dwelling may be evident, but the dwelling may not take place at all. Just creating a space for dwelling, even with all the socio-cultural elements seemingly intact does not ensure that dwelling will occur (Heidegger 1951).

But are there features of virtual locations that inherently make them habitable? Are there social affordances that evoke and sustain habitation? Or is it broader social discourses related to control, power, and social or political influences that pervade these spaces, or a combination of these various elements. It can be argued that we have always had informational selves that we project differing versions of, in different contexts, and for varying purposes. It is not so much that our informational identities or profiles stem from social media, but that social media is another iteration in the presentation of our informational self (Koopman 2019). However, he does contend that the formatting of these more contemporary digital versions does present data points that structure subjects for consumer marketing. These targeted data points, allied with the potential for panoptic surveillance that some apps or platforms engender, open up potential for subtle, seemingly unobtrusive consumer or political manipulation.

We might also consider parallel or synergistic platforms or apps and ways that these interact and are mutually influential such as using moodle as a formal e-learning platform supplemented by twitter as an informal one (Alghamdi 2019). We now reflect on a more recent rendition of spaces that has emerged through the examination of a digital environment, to subsequently consider the relationship with Heidegger's insights. 
Although Heidegger might see digital technologies as structuring the nature of the educative experience in a particular way, not allowing what Lambeir (2002) described as time to be troubled or enraptured by concepts or other phenomena, it also proffers potential for openness. It disintegrates what have historically been relatively stable political or judicial borders (Lambeir 2002). This gestures towards a new openness for individuals as versions and perceptions of truth they engage with are not determined by for instance, political dictators. Digital technologies specifically related to the Internet offer the chance to facilitate the developing societal transition to openness (Peters 2009). He contends that it 'offers ontologically the means to become open: being open and open being' (Peters 2009: 5).

If used appropriately in a pedagogical sense, the use of digital technologies changes the type of thinking from linear or authorial thinking by enabling the creation of personalized stories. This challenges the notion of using digital technologies to practice core skills in a real-life context to the actual creation of versions of the learner's thinking and processing of that thinking. As Bonnett (2002) contends in his interpretation of Heidegger's theorizing of education: 'The essence of technology is a frame of mind or way of seeing things.' He described a 'mode of revealing' and that it 'expresses a certain relationship between human beings and the world' (Bonnett 2002: 191). He saw Heidegger's account of learning as a highly demanding and a participatory affair which requires the learner being engaged fully (Bonnett 2002). This description of an engaging exploration into the unfamiliar led by the student's interests and wonderings fits well with what educationalists envisage for the pedagogical use of digital technologies. However, it might manifest in terms of both classroom pedagogy and the potential to subvert openness within society (e.g., influencing political processes through social media).

New technologies can lend themselves to the learner being artistic. A pedagogical and communication medium might enable perceived and actual open-minded discovery (Bonnett 2002). Used for this type of expression it draws the experience closer to Heidegger's notion of dwelling poetically. It can offer students opportunities to reveal their concerns and delights (Bonnett 2002). Perhaps, this is one thing that influences where we dwell and what locations we are drawn to, and what might also draw likeminded or oppositional thinking. These locations might also be affinity spaces in the sense that Gee articulates them.

The next example illustrates the creation of entities through interactions about creative processes that also indicate students' insights into those processes. If the apps change (which is inevitable) it is not necessarily the nature of the apps or their affordances that matter so much as the potential to facilitate more generic aspects such as multi-media engagement and collaborative construction.

Vignette 4: Student blogs. The following vignette reports on an aspect of a 2-year project related to the building of teacher knowledge when using apps in the teaching and learning of mathematics. While the researchers identified the initial themes and codes, refinement occurred through joint critical reflection between teacher practitioners and academic researchers in research meetings. Two of the 
themes identified in the research meetings were socio-material assemblages and collaboration (Calder and Murphy 2018). Data from student focus group interviews and individual blogs show the ways that socio-technological assemblages and facilitation of student collaboration may help to understand the students' interactions with mathematical ideas, and what influenced the students to inhabit some of the digital learning spaces. Data from the student blogs referred to the use of dynamic multi-modal representations, with students also referring to mediation through programming and the use of different pedagogical media. The use of programming apps with Sphero robots made strong physical and visual connections too, especially in geometry. At times, the connection between hand movements and movement of the Sphero was mediated by the app.

(From the blogs)

Tickle helped me programme robots to draw triangles.

We used this app (Tickle) to learn about making shapes, angles and vertices.

The use of screen casting to record their solution strategies was a key feature. In the interview data, the students talked of videoing themselves doing maths, and recording their working. As one wrote in their blog,

it's just like making a movie for maths.

Hence, students were indicating the multi-modal representational affordances of the screen-casting app. The opportunity to record their voices as well as writing and drawing seemed important to the students. For example, one student commented that it was:

...hard to explain without writing down. You can write it down as well as explaining it while you are recording.

The use of multiple modes simultaneously supported this student in expressing his thinking. The opportunity to pause and edit recordings also appeared to support students to express their thinking. For instance,

the cool thing is that you can pause it and then think about what you are going to do.

The responses from students in the interviews and the blogs suggested further viewpoints in relation to the use of multiple modes in expressing and creating their thinking, and peer collaboration in sharing ideas and in exploring or working with new knowledge. While apps are constantly in modification, with new ones regularly becoming available, generic elements such as having opportunity to work in a multi-modal environment and collaboration appear to facilitate engagement. Within the school context, this suggests habitation rather than visiting. Also, students recording their voices as well as writing and drawing, are creative processes. Creativity is linked to habitat. Social and 


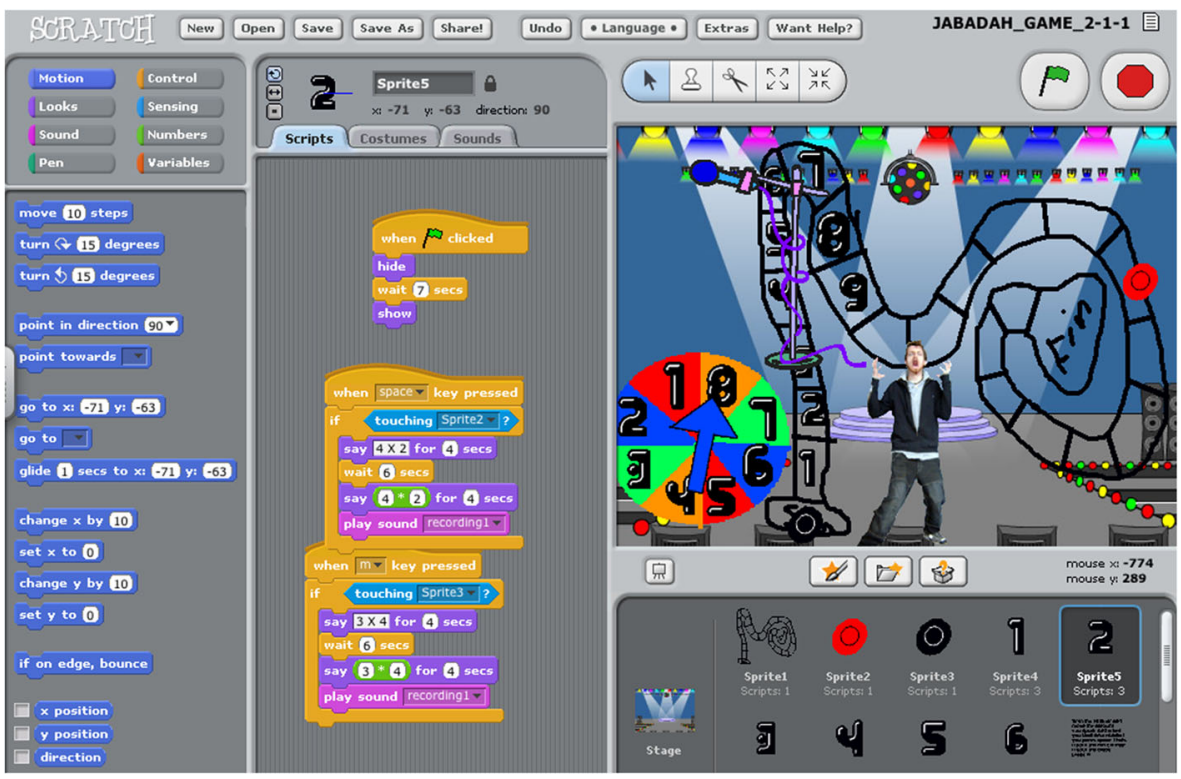

Fig. 4 One group's workspace as their game is in development

cultural experiences always condition our situation (Gallagher 1992), and thus the perspective from which our interpretations are made. Learners engage with the task through a cyclical process of interpretation, engagement, reflection and re-interpretation. Other researchers have perceived learning as an iterative process of re-engagements of collectives of learners, media, and other environmental aspects, with phenomena (e.g., Borba and Villarreal 2005).

The next example reports on 9 and 10 year old students' collaborative problem solving as they used the coding app Scratch to create mathematical games for their 6year-old 'buddy' class.

Vignette 5: Scratch. In this vignette, the students worked in pairs, which were self-selected. Over a 2 -week research period, the students wrote daily blogs articulating their progress and reflections, students and the teacher were interviewed, and classroom observations (both written and photographic) were recorded. The pairs were given the same design brief: To design and build a mathematics game suitable for facilitating the number understanding of their Year 1 (aged 5-6) buddies. The students interviewed their Year 1 buddy class partners, two students with two buddies, and consulted the Year 1 teacher regarding appropriate mathematics concepts and activities that the children were familiar with. This helped determine the nature of the games they would devise (see Fig. 4). While the pairs worked to the same brief, their solutions varied in both content and approach. 
At each juncture, the response to their engagement with the task modified their approach and enabled them to re-engage from their modified perspective. Thus, their thinking evolved, and the games became more refined.

James: We can't get it to go forever - we'll need to explore different loops.

Don: What if we glide until it points to the direction?

James: We can point towards.

Don: Yes, and what about exploring the use of 'sense'?

When they reached a point of uncertainty, they used a 'predict and check' approach. They reflected on the outcome, before refining their evolving script. Again, this was an individual group's creative response.

Their thinking evolved through the problem-solving process. The challenge of the problem-solving process was evident in the blog from another group:

We are trying to figure out how to use a gravity effect and how to use the variables. We are finding it challenging to make our character Jetman jump in the air without spinning 15 degrees.

The project considered that learning is mediated by language and the use of tools. Not only does the dialogue of the teacher and the learners in the classroom act as a mediator, the app itself acts as a mediating tool. The learner's preconceptions of the pedagogical media, in conjunction with the affordances of the media, promote distinct pathways in the learning process. The learner's interaction with the mathematics through the device will influence the ways that the mathematical ideas develop and are understood. This pedagogical device is more than an environment. It is imbued with a complexity of relationships evoked by the users, and the influence of underlying discourses. There is also a relationship between the material environment that the students and their buddy class inhabited and the digital world in which the games were created and enacted. The students received oral feedback from their buddy class after playing the games as well as digital feedback from the coding process. This also illustrates the earlier point regarding a postdigital perspective, where the differences between physical activity and spaces, and digital pedagogical media become more entangled and less distinct (Calder and Murphy 2018). In this vignette, this was notable in the creativity aspect.

There is creativity evidenced through the individual designs and ideas collaboratively negotiated by the pairs of students, but also through each pair's interpretation of the task, their buddy pupils understanding and interests, and through negotiated feedback from both social and technological feedback. The ways of thinking were participatory and highly engaging (Bonnett 2002), with the task and the associated problem-solving elements, led by the students' interests as they ventured into unknown concepts and processes. 


\section{Conclusion}

We now draw together our discussion by considering ideas related to Gee's (2005) notion of affinity spaces and Heidegger's (1951) notion of dwelling. We consider where there is a weaving or synthesis, and where our interpretation might cleave or contrast. In this way, we offer further insights into their respective positions. Both Gee and Heidegger articulate positions where people are central. For Heidegger, dwelling was based on the relationship of people to locations and space, while Gee's affinity spaces were locations where people congregate, allowing for ebbs and flows of engagement and intensity. Both also acknowledge that these might be physical or digital - something that we have indicated by using a mixture of in our illustrations and vignettes.

However, while Gee saw affinity spaces as important contemporary configurations that might initiate participation and collaboration, he also advocated that content was a central element of affinity spaces. For Heidegger the relationship between people and space, rather than the content in the space, was crucial. For Gee, content is the key feature that initiates engagement and occupation, while for Heidegger it seems that location is the key. We would suggest that there is a range of possible interplays between location and content. Bonnett (2002) described technology as more a way of perceiving phenomena with his view that Heidegger (1977: 13) suggested a 'mode of revealing' rather than the content being the initiator.

They both did interpret or were interpreted (e.g., Lambeir 2002) as envisaging digital technologies structuring learning in particular ways, with a blend of functional and aesthetic elements, and of social and technical elements. This resonates with Calder and Murphy's (2018) notion of a socio-material assemblage when using digital technologies in educative settings. The screen-casting feature of iPads was also used to illustrate the creative and personalized nature of the learning through this process (Calder and Murphy 2018). As one student in their study said: 'It was like making a movie for maths.' This is akin to one of Bonnett's (2002) interpretations of Heidegger's dwelling, which he saw as creating versions of the learner's thinking and processing. Heidegger did not acquiesce to digital technology's positive influence on pedagogy, and his position on habitation was articulated in 1951, prior to the recent global uptake of digital technology and social media in a breadth of cultural settings. Nevertheless, we contend that his theorizing helps to facilitate the unpacking, interpretation, and better understanding of digital spaces and why people might dwell in them. Likewise, we hope that our discussion helps extend the interpretation of Heidegger's theorizing to some small extent.

Personalization also offered the opportunity to play with possibilities; to be inquisitive (Bonnett 2002). Gee (2005) identified the facilitation of deep thinking through affinity spaces, also seeing learning through digital technologies as a personal, unique, and social trajectory. Allied to both these are the influence of affordances, the opportunities and constraints that emerge through the complementarity of the user and the environment (Gibson 1977). What are the affordances of the places that we inhabit rather than visit? Perhaps they are more social, involving interaction, but with autonomy, and self-selection? We have addressed some of these aspects directly, such as the social aspects and interaction, autonomy and self-selection. Part of our discussion of personalization is 
more contestable due to the affordances and interplay of the digital technologies or social network spaces. The layers of control and associated power that might pervade these spaces are not transparent. Yet, Lambeir's (2002) and Peters' (2009) proposition that social media are influential in opening up space for openness (and across borders) offers further optimism.

We are also wondering what this means for relatively new technologies such as augmented and virtual reality. How might they create personal spaces in perhaps more complex and influential ways? We also might assume that they would be accompanied by unique ontological and epistemological positions, and a spectrum of distributed cognition. More sophisticated artificial intelligence is also emerging rapidly. So how might collective or synchronized intelligence, across varying blends of material, human and digital assemblages, create new dwellings, spaces and learning? We contend that as the composition of these assemblages varies to create a range of spaces that might be visited or inhabited, they become new, synthesized entities, with the distinction between the contributing material, human or digital elements blurring.

In this paper, we have used an eclectic range of vignettes to illustrate various positions or elements in our discussion, while the nature of the transitions both within and between spaces is also indicated. This suggests that the power is not sited solely with the digital technology or their associated locations. If there is a creative or individual element, then some human choice is inherent. While, as stated previously, there seems to be a symbiotic relationship between the user and the digital technology (Hoyles and Noss 2003), with each mutually influential.

So we return to our question about what happens when we go online, interact and leave our digital footprints? More specifically we were concerned with the implications when teachers and their students go online. Our examples allowed us to sketch a movement within and between spaces and how this necessitates choicemaking and understanding mutually-influencial engagements between users and digital spaces.

This resonates with Dreyfus (2000: 324) who stated that: 'Being able to open and dwell in a number of worlds, we argue, is as much integrity as a human being needs in order to resist being a flexible resource.' While Dreyfus in his general positioning is not affirming of digital technologies being conducive to learning as pedagogical media, we have illustrated that they open up alternative spaces that participants can dwell in or visit, and can transition between. This suggests the potential for new ways to learn through the different pedagogical media, which we illustrate through the various research vignettes. While making sense of these new ways of learning, we contend that Heidegger and Gee facilitate ways for us to consider, and to better interpret and understand, the engagements between users and digital spaces.

Funding Open access funding provided by University of Graz.

Open Access This article is licensed under a Creative Commons Attribution 4.0 International License, which permits use, sharing, adaptation, distribution and reproduction in any medium or format, as long as you give appropriate credit to the original author(s) and the source, provide a link to the Creative Commons licence, and indicate if changes were made. The images or other third party material in this article are included in the article's Creative Commons licence, unless indicated otherwise in a credit line to the material. If material is not included in the article's Creative Commons licence and your intended use is not permitted by statutory regulation or exceeds the permitted use, you will need to obtain permission directly from the copyright holder. To view a copy of this licence, visit http://creativecommons.org/licenses/by/4.0/. 


\section{References}

Alghamdi, F. (2019). The role of social media in developing online learning communities. PhD dissertation. Exeter: University of Exeter. https://ore.exeter.ac.uk/repository/bitstream/handle/10871/39495/ Alghamdi,F.pdf?sequence=2. Accessed 1 November 2020.

Binder, J., Howes, A., \& Sutcliffe, A. G. (2009). The problem of conflicting social spheres: effects of network structure on experienced tension in social network sites. In Proceedings: CHI '09 human factors in computing systems, Boston (pp. 965-975). New York, NY: ACM Press. https://doi.org/10.1145/1753846. 1754177.

Bonnett, M. (2002). Education as a form of the poetic: A Heideggerian approach to learning and the teacherpupil relationship. In M. A. Peters (Ed.), Heidegger, education, and modernity (pp. 229-243). Lanham: Rowman and Littlefield.

Borba, M. C., \& Villarreal, M. E. (2005). Humans-with-media and the reorganization of mathematical thinking: Information and communication technologies, modeling, experimentation and visualisation. New York: Springer.

Calder, N. S., \& Murphy. (2018). How might apps reshape the mathematical learning experience? In N. S. Calder, K. Larkin, \& N. Sinclair (Eds.), Using mobile technologies in the teaching and learning of mathematics (pp. 31-50). Dordrecht: Springer. https://doi.org/10.1007/978-3-319-90179-4_1.

Dreyfus, H. (2000). Responses. In M. Wrathall \& J. Malpas (Eds.), Heidegger, authenticity and modernity: essays in honor of Hubert L. Dreyfus. Cambridge: MIT Press.

Gallagher, S. (1992). Hermeneutics and education. New York: State University of New York Press.

Gee, J. P. (2005). Semiotic social spaces and affinity spaces. From the age of mythology to today's schools. In D. Barton \& K. Tusting (Eds.), Beyond communities of practice: language, power and social context (pp. 214-232). Cambridge: Cambridge University Press. https://doi.org/10.1017/CBO9780511610554.012.

Gibson, J. J. (1977). The theory of affordances. In R. Shaw \& J. Bransford (Eds.), Perceiving, acting, and knowing: toward an ecological psychology (pp. 67-83). Hillsdale, NJ: Lawrence Erlbaum.

Heidegger, M. (1951). Building, dwelling, thinking. Visual Culture: Critical Concepts in Media and Cultural Studies, 3, 66-76.

Heidegger, M. (1977). The question concerning technology and other essays. Trans. and Introduction W. Lovitt. New York: Harper \& Row.

Hoyles, C., \& Noss, R. (2003). What can digital technologies take from and bring to research in mathematics education? In A. J. Bishop, M. A. Clements, C. Keitel, J. Kirkpatrick, \& F. Leung (Eds.), Second international handbook of mathematics education (pp. 323-349). Dordrecht: Kluwer Academic. https:// doi.org/10.1007/978-94-010-0273-8_11.

Ingold, T. (2005). Epilogue: Towards a politics of dwelling. Conservation and Society, 501-508. http://www. conservationandsociety.org/text.asp?2005/3/2/501/49324. Accessed 5 November 2020.

Koopman, C. (2019). Redesign: data's turbulent pasts and future paths. In how we became our data: A genealogy of the informational person. Chicago: University of Chicago Press. https://doi.org/10.7208/ chicago/9780226626611.001.0001.

Lambeir, B. (2002). Comfortably numb in the digital era: Man's being as standing-reserve or dwelling silently. In M. A. Peters (Ed.), Heidegger, education, and modernity (pp. 103-121). Lanham: Rowman \& Littlefield.

Meyer, B. (2015). iPads in inclusive classrooms: Ecologies of learning. In P. Isaias, J. M. Spector, \& D. Ifenthaler (Eds.), E-learning systems, environments and approaches theory and implementation (pp. 2537). Dordrecht: Springer. https://doi.org/10.1007/978-3-319-05825-2_3.

Otrel-Cass, K. (2019). Hyperconnectivity and digital reality: an introduction. In K. Otrel-Cass (Ed.), Hyperconnectivity and digital reality (pp. 323-349). Cham: Springer.

Otrel-Cass, K., \& Andrule, K. (2015). Ontological assumptions in techno-antropological exploration of online dialogue through information systems. Techné: Research in Philosophy and Technology, 19(2), 125-142. https://doi.org/10.5840/techne201571031.

Peters, M. A. (2009). Editorial: Heidegger, phenomenology, education. Educational Philosophy and Theory, 41(1), 1-6. https://doi.org/10.1111/j.1469-5812.2008.00516.x.

Richter, S. (1995). The beauty of building, dwelling, and monk: aesthetics, religion, and the architectural qualities of jazz. African American Review, 29(2), 259-268. https://doi.org/10.2307/3042304.

Spalding, N. J., \& Phillips, T. (2007). Exploring the use of vignettes: from validity to trustworthiness. Qualitative Health Research, 17(7), 954-962. https://doi.org/10.1177/1049732307306187. 
Treem, J. W., \& Leonardi, P. M. (2012). Social media use in organizations: exploring the affordances of visibility, editability, persistence, and association. SSRN Electronic Journal, 36(36), 143-189. https://doi. org/10.1080/23808985.2013.11679130.

Welsch, W. (2014). Schiller revisited: "beauty is freedom in appearance" aesthetics as a challenge to the modern way of thinking. Contemporary aesthetics, 12(1), 16. http://hdl.handle.net/2027/spo.7523862. 0012.016. Accessed 5 November 2020.

Wenger, E. (1998). Communities of practice: learning as a social system. Systems Thinker, 9(5), 2-3. https:// thesystemsthinker.com/communities-of-practice-learning-as-a-social-system/. Accessed 5 November 2020 .

Wenger, E. (2010). Communities of practice and social learning systems: The career of a concept. In C. Blackmore (Ed.), Social learning systems and communities of practice (pp. 179-198). London: Springer. https://doi.org/10.1007/978-1-84996-133-2_11.

Willacy, H., West, A., Murphy, C., \& Calder, N. (2017). Personalised learning with mobile technologies in mathematics: an exploration of classroom practice. Teachers and Curriculum, 17(2), 77-84. https://doi. org/10.15663/tandc.v17i2.173.

Williams, J., Cowie, B., Khoo, E., Saunders, K., Tylor, S., \& Otrel-Cass. (2013). Implementing e-networksupported inquiry learning in science. Research Information for Teachers, 3, 11-19. https://doi.org/10. 18296/set.0351. 